\title{
Nanodispenser for attoliter volume deposition using atomic force microscopy probes modified by focused-ion-beam milling
}

\author{
A. Meister and M. Liley \\ Swiss Center for Electronics and Microtechnology (CSEM SA), CH-2007 Neuchâtel, Switzerland \\ J. Brugger \\ Swiss Federal Institute of Technology (EPFL), CH-1015 Lausanne, Switzerland \\ R. Pugin and H. Heinzelmann ${ }^{\text {a) }}$ \\ Swiss Center for Electronics and Microtechnology (CSEM SA), CH-2007 Neuchâtel, Switzerland
}

(Received 29 June 2004; accepted 8 November 2004)

\begin{abstract}
In this letter, we describe the on-demand dispensing of single liquid droplets with volumes down to a few attoliters and submicrometric spacing. This dispensing is achieved using a standard atomic force microscope probe, with a $200 \mathrm{~nm}$ aperture at the tip apex, opened by focused ion beam milling. The inside of the tip is used as reservoir for the liquid. This maskless dispensing, realized in ambient environment, permits the direct creation of droplet arrays. Nanoparticles, suspended in the liquid, were organized on a surface. (C) 2004 American Institute of Physics.
\end{abstract}

[DOI: $10.1063 / 1.1842352]$

Originally designed for the study of surfaces at high resolution, scanning probe microscopes (SPM) are being increasingly used as active tools in surface engineering, particularly in the deposition and patterning of material. This approach to rapid prototyping bypasses many of the limitations associated with photoresist-based lithography. The shadow mask technique combined with an atomic force microscope (AFM) probe allows one to deposit sub-100 nm metallic nanowires, ${ }^{1}$ micropipettes handled with an SPM are used to deliver liquid, ${ }^{2,3}$ gas $^{4}$ or biomolecules, ${ }^{5}$ and inked AFM tips can serve as pens on a nanometric scale. ${ }^{6,7}$ To circumvent the serial manner of writing, the production of multiple cantilever probes permits parallelization of the process. ${ }^{8,9}$

In this letter, we describe nanoscale dispensing (NADIS) with a probe that consists of an AFM tip designed with a small opening at its apex. This method permits the ondemand deposition of small single droplets in ambient environment at a predetermined location on a sample (Fig. 1). The controlled dispensing of a small amount of liquid is of great interest, both for the economical use of the liquid itself, and because of the high droplet densities that can be achieved. In most of the applications foreseen, the liquid will transport other entities such as biological molecules for the creation of miniaturized high-density bioarrays. Earlier experiments ${ }^{10}$ allowed us to deposit droplets in the femtoliter range. Our search for ever smaller droplets pushed us to develop the attoliter droplet dispensing described here.

The dispenser was created by modifying a commercially available silicon nitride (SiN) AFM probe. ${ }^{11}$ Focused ion beam (FIB) milling ${ }^{12}$ was used to open a $200-n m$-wide aperture at the tip apex [Fig. 2(a)]. In addition, a loading area for the liquid was created using FIB to locally remove the reflective gold layer covering the upper part of the cantilever. The gold layer was removed around and inside the hollow tip, leaving an unexposed SiN surface. While the remaining a) Author to whom correspondence should be addressed; electronic mail:
harry.heinzelmann@ @csem.ch gold was made hydrophobic by chemical treatment, ${ }^{13}$ the bare SiN remained hydrophilic. The hydrophilichydrophobic contrast serves as a virtual wall for confinement of the liquid inside the loading area [Fig. 2(b)]. The shape of the loading area must be judiciously chosen so as to permit the liquid to reach the hollow tip. A smooth shape in the vicinity of the tip is more suitable, since the surface tension of the liquid attempts to keep the droplet in a spherical shape. The outer part of the tip can also be made hydrophobic using double-sided gold-coated cantilevers.

Droplet dispensing was carried out on polished monocrystalline $\mathrm{Si}$ wafers with a native oxide layer (roughness $R \mathrm{~ms}<0.2 \mathrm{~nm}$ over a $10 \mu \mathrm{m}^{2}$ area). The wafers were stored in ambient conditions, and had a fairly low surface energy (hydrophobic). The contact angle of glycerol was $64^{\circ}$ for the advancing contact line and $48^{\circ}$ for the receding one (the sample was previously cleaned in an ultrasonic bath with acetone and ethanol, and rinsed with Millipore water). Oxygen plasma treatment of the sample, or immersion in Piranha solution and copious rinsing with Millipore water, increased the surface energy. The glycerol advancing contact angle was then less than $10^{\circ}$ (measured directly after the treatment). On storing such a sample in an ambient environment, it loses its hydrophilic character within a few days.

All experiments were realized on standard SPM equipment ${ }^{14}$ in an ambient environment (temperature: $21-23{ }^{\circ} \mathrm{C}$, relative humidity: $45 \%-50 \%$ ). Prior to deposition, the liquid was loaded onto the loading area using a tapered glass pipette, with hand driven micromanipulators.

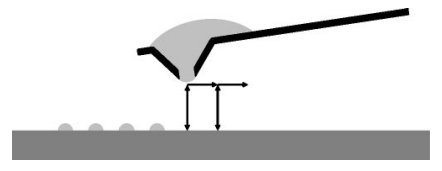

FIG. 1. Sketch of the nanoscale dispensing (NADIS) principle. A hollow AFM tip with an aperture at its apex is loaded with liquid. By bringing the tip briefly into contact with the sample, some liquid is transferred by capillarity onto the surface (no external pressure is applied to the liquid). A SPM drives the probe, so that high accuracy is provided in the positioning of the droplets. 


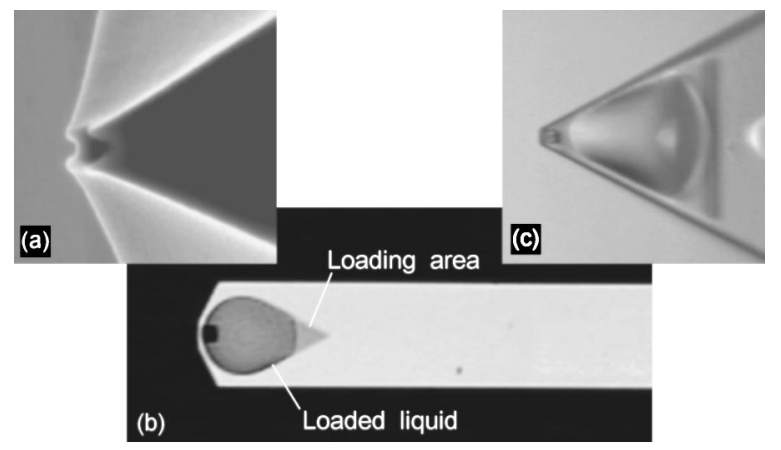

FIG. 2. (a) Scanning electron micrograph $(2 \times 2 \mu \mathrm{m})$. Tip with a $200 \mathrm{~nm}$ aperture at its apex. (b) Optical micrograph $(200 \times 100 \mu \mathrm{m})$. Upper face of a NADIS probe with the loading area partially filled with liquid. (c) Optical micrograph $(100 \times 100 \mu \mathrm{m})$. Poorly designed loading area. The surface tension of the liquid prevents the liquid flowing towards the acute angle of the triangular-shaped loading area and reaching the hollow tip.

The liquid filled the hollow tip and the aperture by capillarity. Capillarity also prevents the flow of the liquid through the aperture until contact is made with the sample. The amount of liquid loaded can be estimated from the shift of the cantilever resonance frequency. ${ }^{15}$ Typical loading volumes are some 100's of femtoliters. For practical use, liquids with low evaporation rates are most suitable, such as glycerol or tetraethylene glycol. ${ }^{16}$ The probe was driven in contact mode using the NanoScript ${ }^{\mathrm{TM}}$ lithography language. After a dispensing experiment, the tip could be cleaned (immersion in solvent, oxygen plasma, chemical treatment) and reused.

The volume of a deposited droplet was determined by its size (diameter and height). The lying droplet is assumed to be a spherical cap. The height was determined by the snap-in position on a cantilever-deflection versus probe-sampledistance curve, recorded onto the droplet directly after its deposition. Since the droplets were too small to be measured optically, their diameter was estimated as the smallest distance between two noncoalescing droplets in an array with diminishing pitches. If during the deposition the liquid meniscus around the tip touches the previous deposited droplet, the latter will merge into the meniscus by the surface tension.

Using the nanodispenser (single-sided coated cantilever), glycerol droplets were dispensed onto a hydrophobic sample to form an array. The time needed to dispense one droplet was about $0.5 \mathrm{~s}$ and the droplet height was measured to be $100 \mathrm{~nm}$. After the full evaporation of the droplets, the area was imaged by tapping mode AFM. An array of dots ( $\sim 20 \mathrm{~nm}$ in height, $\sim 70 \mathrm{~nm}$ in diameter) was clearly visible [Fig. 3(a)]. We suppose that the dots consist of nonvolatile residues present in the glycerol, since an identical experiment, performed using the probe without liquid, resulted in a clean, bare surface. The dot spacing corresponds to the programmed spacing for the dispensing, i.e., $1 \mu \mathrm{m}$. The micrograph reveals that a large number of almost identical dots can be patterned. Since the initial droplet diameter must be less than the dot spacing, the maximum value for the droplet volume was about 40 al. One loading of the probe permitted us to dispense more than a 1000 such droplets onto a hydrophobic sample, with still some liquid remaining in the loading area.

In additional experiments, we deposited glycerol containing $20 \mathrm{~nm}$ polystyrene nanoparticles ${ }^{17}$ onto a more hydrophilic sample (advancing glycerol contact angle measured Downloaded 17 Dec 2004 to 128.178.153.159. Redistribution subject

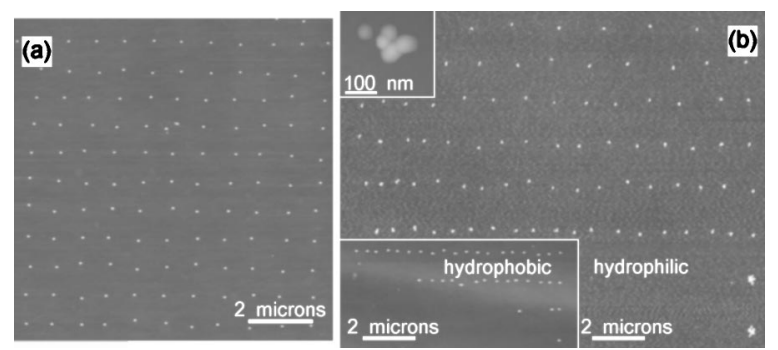

FIG. 3. Topographic tapping AFM micrographs. (a) Array of dots made of the nonvolatile residue formed after the evaporation of glycerol droplets deposited by NADIS. (b) Array of $20 \mathrm{~nm}$ polystyrene nanoparticles dispensed by NADIS. The dots consist of a few beads (upper inset). On the hydrophilic surface, the smallest dot spacing achieved is $800 \mathrm{~nm}$, whereas on the hydrophobic substrate, it is about $410 \mathrm{~nm}$. The misalignment of the dots is due to the hysteresis of the SPM piezo-scanner, and to pinning effects of the three-phase contact line (droplet border) during evaporation.

to be $35^{\circ}$ ). The droplet array, with diminishing pitch from line to line, was dispensed from a double-sided gold-coated probe. After evaporation of the glycerol, the nanoparticles were imaged by tapping AFM [Fig. 3(b)]. The first six lines are easily discernible. The last two lines are missing, except for the last dot in each line, where the number of particles is higher compared to the dots in the first lines, due to the coalescing of the liquid droplets. The value of the droplet diameter must lie between the average spacing in the last recognizable line $(800 \mathrm{~nm})$ and the expected spacing in the first missing line $(720 \mathrm{~nm})$. A similar experiment was done onto a hydrophobic substrate, where the droplet diameter was measured to be between 410 and $370 \mathrm{~nm}$ [lower inset in Fig. 3(b)]. In the latter, the droplet height was measured to be $70 \mathrm{~nm}$, resulting in a deposited volume of about 5 al per droplet.

The amount of deposited liquid is mainly defined by the size of the liquid meniscus between tip and sample before the probe withdrawal. In a hydrostatic case, the geometrical shape of the interface air-liquid has to fulfill the pressure equilibrium given by $\gamma\left(1 / R_{1}+1 / R_{2}\right)+\rho g z=$ const. The first term corresponds to the local Laplace pressure, ${ }^{18}$ where $\gamma$ is the liquid surface tension, and $R_{1}$ and $R_{2}$ the radii ${ }^{19}$ of the local mean curvature at the liquid-air interface. The second term is due to gravity and can be neglected for small dimensions, so that the equilibrium condition reduces to a constant curvature over the entire liquid-air interface, at the meniscus as well as over the loading area. Otherwise, a pressure gradient induces a flow of liquid until the equilibrium is reached. Moreover, the shape of the meniscus is also influenced by the contact angle of the liquid with the sample or the outer tip wall. Numerical simulations (Fig. 4) as well as laboratory tests show that with hydrophilic tip and sample surfaces, no equilibrium is reached until the loading area is almost empty, so that nearly all the liquid flows through the aperture to create a huge meniscus. Nevertheless, on a hydrophilic sample, a tip with a hydrophobic outer wall achieves more suitable conditions. The smallest meniscus is achieved with a hydrophobic sample, permitting the deposition of denser and more numerous identical droplets without reloading of the probe. If the contact time is shorter than that needed to reach the hydrostatic equilibrium, parameters associated with the liquid viscosity can also influence the volume deposited. Shorter contact times will, e.g., favor less deposition of liquid, or increasing the number of contacts to AlP license or copyright, see http://apl.aip.org/apl/copyright.jsp 

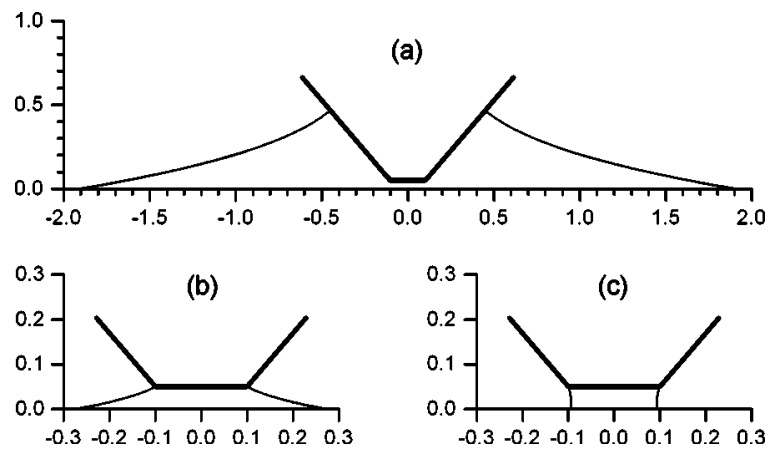

FIG. 4. Theoretical axial symmetric shapes of the liquid meniscus between sample and tip in hydrostatic equilibrium (graph unit: micrometer). The thick line represents the tip, and the horizontal axis the sample surface. The mean curvature radius is identical in all three cases. (a) With hydrophilic tip and sample surfaces, hydrostatic equilibrium results in a huge meniscus. (b) With a tip having a hydrophobic outer wall, the liquid will spread less over the hydrophilic sample. (c) A small meniscus is obtained with a hydrophobic sample.

onto a same position will increase the droplet volume until the equilibrium is reached.

In summary, a modified AFM probe was used to dispense a high-density array of glycerol nanodroplets onto a surface. The dispensing of single droplets, with volume down to 5 al per droplet and spacing below $500 \mathrm{~nm}$, was demonstrated. Using the liquid as a transport medium, patterns could be written with nanoparticles by letting the suspension liquid evaporate. The smallest pitch achieved is obtained on a hydrophobic surface. Further investigations will include the integration of a closed microfluidic channel along the cantilever, to allow the dispensing of water-based solute for biological applications.

The authors wish to thank Philippe Gasser for operating the FIB instrument. The partial support of Top Nano 21, of the National Center of Competence in Research (NCCR) in nanoscale science, and of the Swiss Federal Office for Education and Science (OFES) in the framework of the ECfunded project NaPa (Contract No. NMP4-CT-2003-500120) is gratefully acknowledged. The content of this work is the sole responsibility of the authors.

${ }^{1}$ R. Lüthi, R. R. Schlittler, J. Brugger, P. Vettiger, M. E. Welland, and J. K. Gimzewski, Appl. Phys. Lett. 75, 1314 (1999).

${ }^{2}$ A. Lewis, Y. Kheifetz, E. Shambrodt, A. Radko, E. Khatchatryan, and C. Sukenik, Appl. Phys. Lett. 75, 2689 (1999).

${ }^{3}$ M.-H. Hong, K. H. Kim, J. Bae, and W. Jhe, Appl. Phys. Lett. 77, 2604 (2000).

${ }^{4}$ J. Voigt, B. Reinker, I. W. Rangelow, G. Mariotto, I. Shvets, P. Guethner, and H. Löschner, J. Vac. Sci. Technol. B 17, 2764 (1999).

${ }^{5}$ A. Bruckbauer, L. Ying, A. M. Rothery, D. Zhou, A. I. Shevchuk, C. Abell, Y. E. Korchev, and D. Klenerman, J. Am. Chem. Soc. 124, 8810 (2002).

${ }^{6}$ M. Jaschke and H.-J. Butt, Langmuir 11, 1061 (1995).

${ }^{7}$ R. D. Piner, J. Zhu, F. Xu, S. Hong, and C. A. Mirkin, Science 283, 661 (1999).

${ }^{8}$ P. Vettiger, M. Despont, U. Drechsler, U. Dürig, W. Häberle, M. I. Lutwyche, H. E. Rothuizen, R. Stutz, R. Widmer, and G. K. Binnig, IBM J. Res. Dev. 44, 323 (2000).

${ }^{9}$ P. Belaubre, M. Guirardel, G. Garcia, J. B. Pourciel, V. Leberre, A. Dagkessamanskaia, E. Trévisiol, J. M. François, and C. Bergaud, Appl. Phys. Lett. 82, 3122 (2003).

${ }^{10}$ A. Meister, S. Jeney, M. Liley, T. Akiyama, U. Staufer, N. F. de Rooij, and H. Heinzelmann, Microelectron. Eng. 67-68, 644 (2003).

${ }^{11}$ Olympus OMCL-RC800. Cantilever width: $40 \mu \mathrm{m}$, length: 100/200 $\mu \mathrm{m}$, spring constant: $\sim 0.8 / 0.1 \mathrm{~N} / \mathrm{m}$.

${ }^{12}$ Dual beam FIB (gallium ions and electrons), FEI Company, Hillsboro, OR 97124.

${ }^{13}$ Immersion in hexadecane with $10 \mathrm{mM}$ hexadecanethiol after an oxygen plasma cleaning.

${ }^{14}$ MultiMode and Dimension 3100, Digital Instruments, Veeco Metrology LLC, Santa Barbara, CA 93117.

${ }^{15}$ J. P. Cleveland, S. Manne, D. Bocek, and P. K. Hansma, Rev. Sci. Instrum. 64, 403 (1993).

${ }^{16}$ In ambient environment, the evaporation rate of both liquids is less than $1 \mathrm{al} / \mu \mathrm{m}^{2} / \mathrm{s}$. This value was measured by the time dependency of the cantilever resonance frequency for a loaded probe.

${ }^{17}$ FluoSpheres ${ }^{\circledR}$, catalog No. F-8787, Molecular Probes, Eugene, OR 97402.

${ }^{18}$ Difference of pressure between air and liquid phases induced by the curvature of the interface.

${ }^{19} R_{1}$ and $R_{2}$ are the curvature radii of the surface, belonging to two normal planes perpendicular to the surface at the point of interest. One radius is taken positive if the center of it points inside the liquid. More detail in: A. W. Adamson and A. P. Gast, Physical Chemistry of Surfaces, 6th ed. (Wiley, New York, 1997). 